\title{
¿LÍDERES SEXISTAS? UN ESTUDIO SOBRE LA INFLUENCIA DEL SEXISMO EN LOS ESTILOS DE LIDERAZGO
}

\section{SEXIST LEADERS? A STUDY ON THE INFLUENCE OF SEXISM IN LEADERSHIP STYLES}

Carolina Garzón Bello, Dolores Jiménez Domínguez, Inmaculada Juan Moreno, Cristina Luque Oliva, Rosa María Navío García, Mireia Pacheco Arco, María José Pérez Páez. Universidad de Granada

\section{Resumen}

Dos fenómenos psicosociales como son el sexismo y el estilo de liderazgo podrían estar más relacionados de lo que parece a priori. El objeto de este estudio es analizar cómo se relacionan ambas variables. Se espera que se revelen algunas de las diferencias existentes entre los distintos tipos de sexismo y su relación con los distintos tipos de liderazgo. Para ver la relación entre ambas variables se contó con una muestra de 140 participantes (70 mujeres y 70 hombres), de edades comprendidas entre 18 y 30 años. Los instrumentos empleados fueron el Test de Liderazgo de Kurt Lewin (1940) y la Escala de Sexismo Ambivalente (ASI) de Expósito, Moya y Glick (1998). Los resultados obtenidos mostraron una correlación entre el sexismo hostil con el liderazgo autoritario, y que las diferencias de género en cuanto al sexismo hostil eran mayores en hombres, no mostrando diferencias en el sexismo benévolo.

Palabras clave: estilo de liderazgo, autoritarismo, sexismo

\begin{abstract}
Two psychosocial topics such as sexism and leadership style may be more related than it seems a priori. The purpose of this study is to analyze how they relate both variables. It is expected that this study will reveal some of the differences between the different types of sexism and its relation to the different types of leadership. To see the relationship between both variables had a sample of 140 participants (70 women and 70 men), aged between 18 and 30. The instruments used were the Leadership Test of Kurt Lewin (1940), and Ambivalent Sexism Inventory (ASI) of Expósito, Moya and Glick (1998). The results showed a correlation between hostile sexism authoritarian leadership, and gender differences regarding hostile sexism were higher in men, showing no differences in benevolent sexism.
\end{abstract}

Key words: leadership style, authoritarism, sexism

\section{INTRODUCCIÓN}

"Imaginemos, por ejemplo, a un empresario que tuviera a dos aspirantes a la dirección de su empresa: un hombre y una mujer, ambos con una larga trayectoria en la organización y con un currículum semejante. "Elegiré al hombre porque estos son mejores líderes" o "elegiré a la mujer porque ellas tienen más mano izquierda y saben tratar mejor a los subordinados". Posiblemente, realizaría una mejor decisión si analizara más detenidamente las características idiosincrásicas de cada uno de los dos candidatos, los requisitos del puesto de dirección de que se trata, el tipo de empresa, las peculiaridades de los empleados a los que hay que dirigir, etcétera. (Moya, 2003, p. 322). 
En las últimas décadas se han atribuido características específicas a las personas que desempeñan el papel de líder en cualquier organización. Estas están influenciadas por factores biológicos, psicológicos y sociales que moldean a la persona y a la sociedad ejerciendo una relación bidireccional. Pero, ¿qué se entiende por liderazgo? Según Molero (2004) es un proceso de influencia entre el líder y sus seguidores que persigue conseguir las metas de un grupo, organización o sociedad.

Existen tres estilos de liderazgo, según Lewin (1939): estilo autoritario (caracterizado por ser directivo y hacer énfasis en el distanciamiento social, la determinación y control del trabajo a realizar), estilo democrático (hace énfasis en el trabajo grupal, donde se tiene en cuenta la aprobación y participación de todos en la toma de decisiones) y estilo laissez-faire (estilo no directivo, se basa en la otorgación de libertad de acción y la no participación e implicación en actividades del grupo que lidera).

Tradicionalmente se ha destacado el estilo de liderazgo autoritario, asociado a las características de personalidad típicas masculinas: se presenta al hombre (más que la mujer) como figura prototípica de líder. Un estudio de García-Retamero y López-Zafra (2006) concluyó que se manifiesta un mayor prejuicio hacia las mujeres líderes cuando estas desempeñan tareas tradicionalmente masculinas. Sus éxitos se atribuyen a factores externos (suerte), mientras que los fracasos se asocian a factores internos (falta de capacidad). En cambio, los éxitos masculinos son atribuidos a factores internos y los fracasos a factores externos. Estos prejuicios se mantienen cuando no cumplen con la expectativa del rol de líder esperado: en mujeres se espera que sean relacionales por ser una expectativa inherente al rol femenino, e instrumentales, por ser una característica propia de líder (Limón y Rocha, 2012).

Existen diferencias en la atribución del estilo de liderazgo según el género. Al estilo de liderazgo masculino se atribuyen características competitivas, control detallado, razonamiento analítico y con el objetivo de triunfar en las tareas. En cambio, a las mujeres se atribuyen características cooperativas, empatía, menor control de la situación y orientación hacia la calidad de las relaciones (Arteaga y Ramón, 2009).

Esta desigualdad entre sexos se conoce como sexismo, definido como el conjunto de creencias, actitudes y comportamientos perjudiciales hacia un individuo derivado de la percepción negativa del mismo por pertenecer a un sexo determinado (Moya, Glick, Expósito, De Lemus y Hart, 2007). Define dos tipos de sexismo: benévolo y hostil. El primero hace referencia a un conjunto de actitudes dirigidas hacia las mujeres, considerándolas un ser delicado al que hay que proteger y cuidar (tono afectivo positivo) pero limita la capacidad de autonomía en ciertas tareas. El segundo se define como un prejuicio de subordinación de la mujer, antipatía y/o rechazo explícito hacia ella.

Limón y Rocha (2011) encontraron que se atribuyen características de machismo, abuso y acoso hacia la mujer (sexismo hostil) a líderes con estilo autoritario. Ello implica aspectos comunes entre ambos: este tipo de líderes no aceptan las opiniones de los demás, creen estar en una posición de superioridad y no tienen en cuenta puntos de vista ajenos, imponiendo los propios.

Por ello, nuestro objetivo será establecer si existe relación entre el sexismo y los estilos de liderazgo, mediante las siguientes hipótesis:

1) A mayor sexismo mayor estilo de liderazgo autoritario, existiendo una relación mayor con el sexismo hostil que con el benévolo.

2) A menor sexismo mayor estilo de liderazgo democrático.

3) Existen diferencias de género en sexismo y estilos de liderazgo. 


\section{MÉTODO}

\section{Participantes}

Se contó con una muestra escogida al azar de 140 participantes (70 hombres y 70 mujeres), de edades comprendidas entre 18 y 30 años. La muestra procede de Granada, Córdoba y Jaén.

\section{Procedimiento}

Se administraron los cuadernillos a los participantes de forma individual, en un ambiente controlado, con adecuada iluminación, sin ruidos y sin ningún tipo de distractores. Después, se realizó un análisis de datos utilizando el paquete estadístico SPSS.

\section{Instrumentos}

Las pruebas utilizadas han sido el Test de Liderazgo de Kurt Lewin (1939) y la Escala de Sexismo Ambivalente de Expósito, Moya y Glick (1998). El primero evalúa los estilos de liderazgo que pueden presentar los sujetos ante un rol de mando (autoritario, democrático y laissez-faire). Consta de 33 ítems con respuestas de tipo dicotómico (de acuerdo/en desacuerdo). En cuanto al ASI, se evalúa el nivel de sexismo hostil y sexismo benevolente. Este test consta de 22 ítems, que puntúan en una escala de tipo Likert de 0 a 5.

\section{RESULTADOS}

En la Tabla 1 se encuentran los estadísticos descriptivos para la variable "Sexismo". Se observa una media de 2.69 en Sexismo Hostil, con una desviación típica de 1.17. Por otra parte, la media de Sexismo Benévolo es de 2.62, con una desviación típica de 1,14 .

También se incluyen los estadísticos descriptivos para la variable "Estilos de Liderazgo". La media del estilo de liderazgo autoritario es de 0.43 , con una desviación típica de 0.33. La media del estilo democrático es de 0.73 , siendo la desviación típica de 0.20 . Por último, la media del estilo laissez-faire es de 0.46 , con una desviación típica de 0.18 .

Tabla 1. Estadísticos descriptivos del sexismo y estilos de liderazgo.

\begin{tabular}{lcc}
\hline & Media & $\begin{array}{c}\text { Desviación } \\
\text { Típica }\end{array}$ \\
\hline Sexismo Hostil & 2.69 & 1.17 \\
Sexismo Benevolente & 2.62 & 1.14 \\
Estilo Autoritario & .43 & .33 \\
Estilo Democrático & .73 & .20 \\
Estilo Laissez-faire & .46 & .18 \\
\end{tabular}


A continuación, se realiza el análisis de los datos para cada una de las hipótesis planteadas en el estudio:

1) A mayor sexismo mayor estilo de liderazgo autoritario, existiendo una relación mayor con el sexismo hostil que con el benévolo.

Tabla 2: Correlaciones de Pearson entre el sexismo hostil y benévolo y el estilo de liderazgo autoritario.

\begin{tabular}{lccc}
\hline \multicolumn{4}{c}{ Correlaciones de Pearson } \\
\hline & 1 & 2 & 3 \\
$\begin{array}{l}\text { 1. Sexismo } \\
\text { Hostil }\end{array}$ & - & $.242^{* *}$ & $.522^{* *}$ \\
$\begin{array}{l}\text { 2. Sexismo } \\
\text { Benévolo }\end{array}$ & $.242^{* *}$ & - & $.203^{*}$ \\
3. Estilo & & & - \\
Autoritario & $.522^{* *}$ & $.203^{*}$ & \\
\hline${ }^{* *} p<.01{ }^{*} p<.05$ & & &
\end{tabular}

Como podemos observar, existe una correlación positiva entre ambos tipos de sexismo y el estilo de liderazgo autoritario. Así, la correlación entre el sexismo hostil y el estilo autoritario es de 0.522 , siendo dicha relación significativa $(p<0.01)$.

Por otra parte, la correlación entre el sexismo benévolo y el estilo autoritario también es positiva (0.203), con una significación de $p=0.016$.

Tras estos resultados, se observa una mayor relación del estilo autoritario con el sexismo hostil en relación al Sexismo benévolo.

2) A menor sexismo mayor estilo de liderazgo democrático.

A través del análisis de los datos, se observa una correlación negativa significativa entre el sexismo hostil y el estilo democrático de -0.281. En cuanto al sexismo benévolo, no se ha encontrado una relación significativa con este estilo.

Tabla 3: Correlaciones de Pearson entre el sexismo hostil y benévolo y el estilo de liderazgo democrático.

\section{Correlaciones de Pearson}

\begin{tabular}{lccc}
\hline & 1 & 2 & 3 \\
1. Sexismo Hostil & - & $.242^{* *}$ & $-.281^{* *}$ \\
2. Sexismo Benévolo & $.242^{* *}$ & - & -.002 \\
3. Estilo Democrático & $-.281^{* *}$ & -.002 & - \\
\hline
\end{tabular}

${ }^{* *} p<.01$ 
3) Existen diferencias de género en sexismo y estilos de liderazgo.

En relación al sexismo, se observan diferencias estadísticamente significativas en el sexismo hostil $(p<.01)$, puntuando en mayor medida los hombres en comparación con las mujeres (ver gráfico 1). Sin embargo, no se observan diferencias en el sexismo benévolo (no hay diferencias).
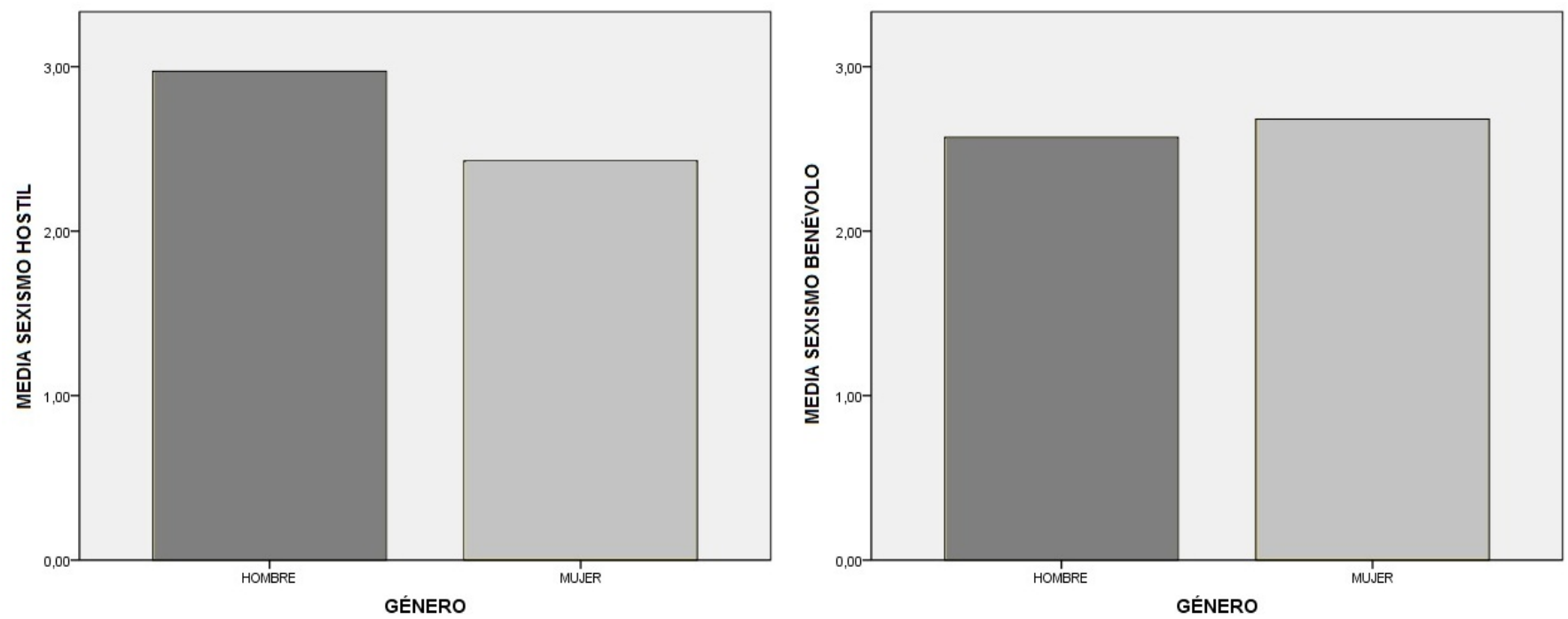

Prueba T para muestras independientes con la variable "Sexismo"

Gráfica 1: Prueba T para muestras independientes con las variables de Sexismo y Género.

Atendiendo a los diferentes estilos de liderazgo, existen diferencias estadísticamente significativas en el estilo de liderazgo autoritario $(p=0.36)$, observándose también una mayor puntuación en hombres que en mujeres. En este caso, no se observan diferencias en el estilo de liderazgo democrático.
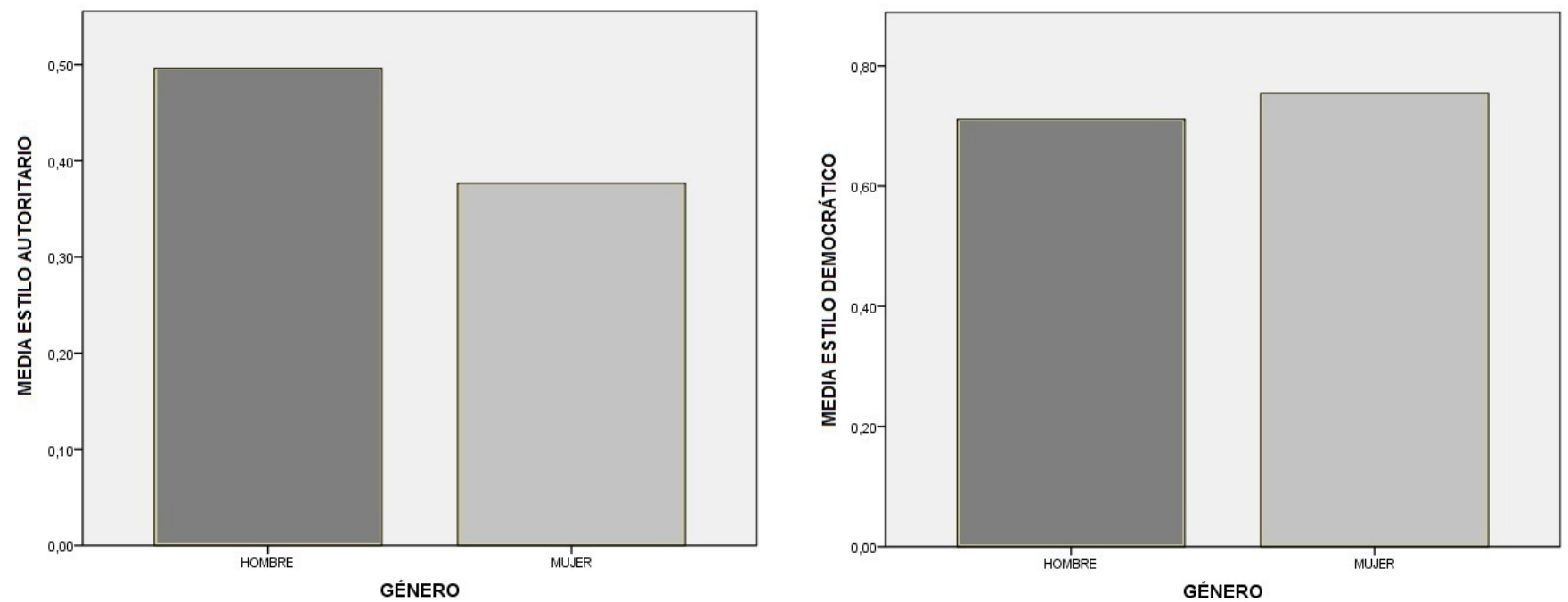

Prueba T para muestras independientes con la variable "Estilos de Liderazgo"

Gráfica 2: Prueba $T$ para muestras independientes con las variables Estilos de liderazgo autoritario y democrático 


\section{DISCUSIÓN}

En la actualidad, en las organizaciones, el rendimiento y satisfacción del empleado en la empresa depende del tipo de liderazgo que se ejerza y el nivel de sexismo que tenga el líder. Por ello, el estudio se centró en determinar si existía relación entre sexismo y liderazgo.

Tras el análisis de los resultados, podemos observar que las hipótesis planteadas se confirman. La primera hipótesis trata de ver la relación entre el sexismo y el estilo de liderazgo autoritario, obteniéndose una correlación positiva entre ambas variables. Este resultado se ve apoyado por Limón y Rocha (2011), defendiendo que el líder autoritario justifica la desigualdad entre sexos y la jerarquía en los puestos de trabajo.

Con respecto a la segunda hipótesis, "A menor sexismo mayor estilo de liderazgo democrático", se ha evidenciado que existe una relación inversa entre estilo de liderazgo democrático y sexismo hostil; sin embargo no se presenta relación con el sexismo benévolo.

El liderazgo democrático resalta valores de igualdad entre trabajadores y toma de decisiones grupal, considerando a todos por igual (Lewin, 1939). Estos valores son totalmente contradictorios al sexismo, que apoya la desigualdad de género y aporta características de superioridad-inferioridad.

Los resultados obtenidos confirmaron que "existen diferencias de género en sexismo y estilos de liderazgo". Los hombres presentan un mayor índice de sexismo hostil que las mujeres, y una media superior de estilo de liderazgo autoritario. Ello se ve respaldado por las características relacionadas al estereotipo del líder masculino, al cual se atribuyen éxitos a factores internos, y cuando actúan como líderes autoritarios se perciben congruentes a la expectativa del rol asumido (García-Retamero y LópezZafra, 2006).

Durante la investigación realizada se propuso una cuarta hipótesis que relacionaba el sexismo con el estilo de liderazgo laissez-faire. Sin embargo, no se pudo analizar ya que no encontramos justificación teórica para dicha hipótesis, lo que supuso una limitación al estudio. Otra limitación es la procedencia de la muestra, recogida en una sola región demográfica (Andalucía), pudiendo influir en los resultados obtenidos, así como la restricción de edad (muestra de población juvenil).

Sería interesante establecer futuros estudios donde se observe la relación entre el origen del estilo de liderazgo y la cultura, los rasgos de personalidad y el tipo de empresa. También podría estudiarse la influencia que tiene el puesto laboral en el estilo de liderazgo que presentan los empleados. Finalmente, podría realizarse otro estudio relacionando sexismo con el estilo de liderazgo laissez-faire.

\section{REFERENCIAS}

Arteaga, A. y Ramón, S. (2009). Liderazgo resonante según género. Multiciencias, 9(3), 289-295.

Expósito, F., Moya, M. y Glick, P. (1998) Sexismo ambivalente: medición y correlatos. Revista de Psicología Social, 13, 159-170.

García-Retamero, R. y López-Zafra, E. (2006). Congruencia de rol de género y liderazgo: El papel de las atribuciones causales sobre el éxito y el fracaso. Revista Latinoamericana de Psicología, 38(2), 245-257. 
Lewin, K. (1939). Experiments in social space. En D. Cartwright (Ed.), Field Theory in social science: selected theoretical papers by Kurt Lewin (pp. 71-83). Nueva York: Harper Torchbooks.

Limón, J. B. y Rocha, T. E. (2011). Creencias y actitudes sexistas de trabajadores en empresas mexicanas: un estudio exploratorio. Psicología Iberoamericana, 19(2), 55-66.

Limón, J. B. y Rocha, T. E. (2012). Prejuicio sexista y atribuciones de éxito o fracaso de líderes: importancia del contexto a evaluar. Enseñanza e investigación en Psicología, 17(2), 329-341.

Molero, F. (2004). El liderazgo. En C. Huici y J.F. Morales (Dir.): Psicología de grupos I. Estructura y procesos, 141-169. Madrid: UNED.

Moya, M. (2003). Sobre la existencia y el origen de las diferencias en el liderazgo entre hombres y mujeres. Revista de Psicología Social, 18(3), 321-325.

Moya, M., Glick, P., Expósito, F., De Lemus, S. \& Hart, J. (2007). It's for your own good: Benevolent Sexism and women's reactions to protectively justified restrictions. Personality and Social Psychology Bulletin, 33(10), 1421-1434. 